03,11

\title{
О скорости термомиграции жидких цилиндрических включений в кристалле в стационарных тепловых условиях
}

\author{
(C) С.И. Гармашов \\ Южный федеральный университет, \\ Ростов-на-Дону, Россия \\ E-mail: garmashov@sfedu.ru
}

Поступила в Редакцию 16 июля 2019 г.

В окончательной редакции 16 июля 2019 г.

Принята к публикации 25 июля 2019 г.

На основе модельных представлений о форме сечения жидкого цилиндрического включения, мигрирующего в кристалле под действием градиента температуры в стационарных тепловых условиях, рассчитана и проанализирована зависимость скорости и формы сечения включения от площади его сечения при разных значениях удельной межфазной энергии, степени ее анизотропии и степени затрудненности межфазных процессов. Показана возможность немонотонности зависимости скорости цилиндрического включения от площади и толщины его сечения.

Ключевые слова: термомиграция; жидкие включения; межфазная кинетика; анизотропия межфазной энергии.

DOI: $10.21883 /$ FTT.2019.12.48538.19ks

\section{1. Введение}

Явление миграции жидкого включения в кристалле под действием градиента температуры (или кратко термомиграция) [1-11] может использоваться как способ получения информации о механизме и кинетике процессов кристаллизации (растворения) $[3,6,7,11]$. Для интерпретации экспериментальных данных по термомиграции возникает необходимость в математической модели этого процесса. В случае включений в виде плоских прослоек, заключенных между двумя кристаллическими пластинами, модель, позволяющая вычислить скорость термомиграции при разных механизмах межфазных процессов, была впервые предложена в работе [3] и в дальнейшем уточнена учетом различных факторов (см. обзор [6]), в том числе нестационарности тепловых условий [7]. Один из основных выводов, вытекающий из модели [3], состоит в том, что межфазная кинетика ограничивает массоперенос в жидкой фазе, в связи с чем скорость миграции убывает с уменьшением толщины жидких прослоек. Вид такой зависимости при малых толщинах прослоек различен для разных механизмов роста (растворения), что может быть использовано по предложению автора [3] для выявления этих механизмов и параметров, характеризующих их. При этом, как отмечается в работе [7], тепловые условия экспериментов по термомиграции должны быть достаточно стационарными и удовлетворять соответствующим критериям.

В случае включений цилиндрической формы возникает необходимость в учете (помимо межфазной кинетики) капиллярных эффектов, то есть изменения условий равновесия фаз в разных точках межфазной границы в зависимости от ее геометрии и анизотропии удельной межфазной энергии. Результатом моделирования термо- миграции в таком случае является не только скорость, но и форма поперечного сечения цилиндрического включения, которая, как следует из экспериментальных данных, приведенных на рис. $1, a$, определяет геометрию перекристаллизованной области. Предложенная в работах [9-11] модель миграции цилиндрического включения достаточно полно учитывает основные факторы, определяющие его скорость и форму - межфазную кинетику, межфазную энергию и их анизотропию.

Цель настоящей работы - анализ зависимостей скорости и формы мигрирующего цилиндрического включения от площади и толщины его сечения, рассчитанных при разной затрудненности межфазных процессов, значениях удельной межфазной энергии и степени ее анизотропии, а также сравнение полученных зависимостей с аналогичными для случая плоских прослоек.

\section{2. Основные допущения используемой модели}

Модель формы сечения цилиндрического включения [9-11], используемая в настоящей работе, основана на балансе потоков в разных точках межфазной границы с учетом капиллярных эффектов и межфазной кинетики в предположении, что градиент температуры в жидкой фазе постоянен во времени, однороден и направлен перпендикулярно плотноупакованным плоскостям кристалла; колебаний температуры нет. Кроме того, считается, что анизотропия удельной межфазной энергии описывается зависимостью $\gamma(\varphi)$ вида [11]:

$$
\gamma(\varphi)=\gamma_{\min }+\left(\gamma_{\max }-\gamma_{\min }\right)|\sin \varphi|,
$$

где $\varphi-$ угол между данной плоскостью кристалла и плоскостью с минимальным значением удельной меж- 

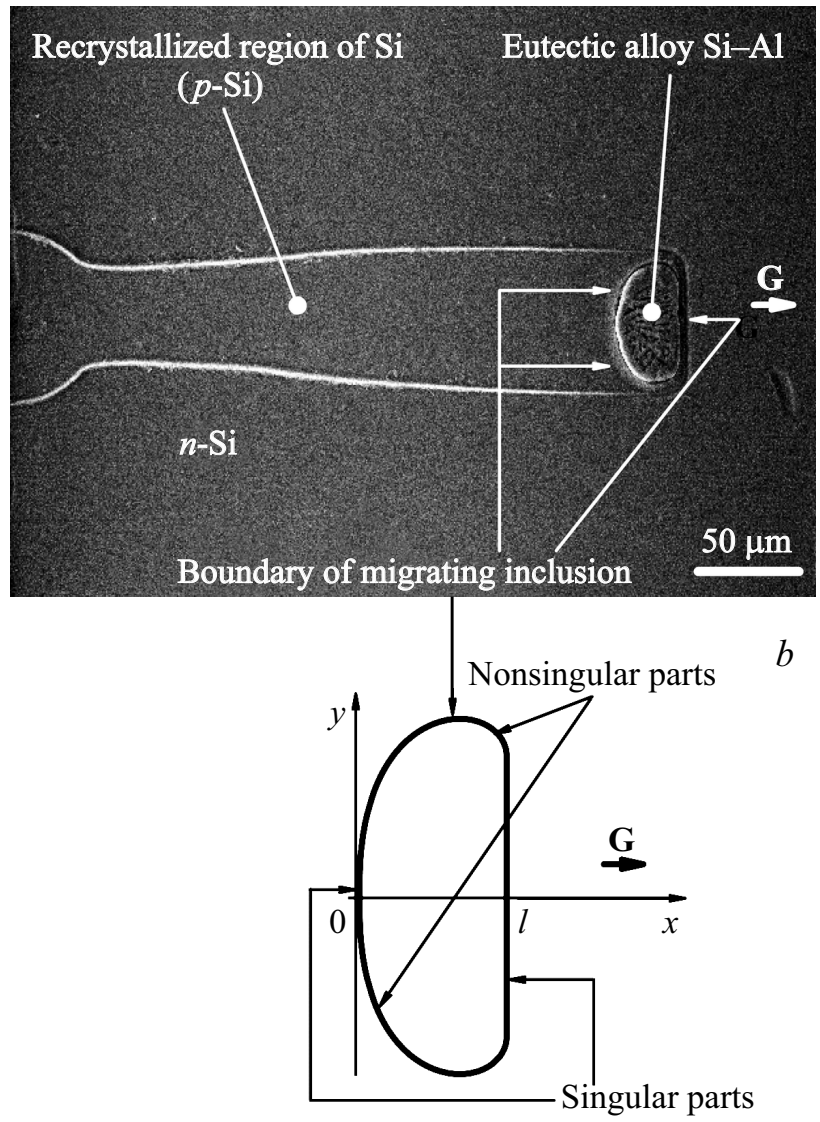

Рис. 1. Вид перекристаллизованной области в пластине кремния после процесса термомиграции жидкого цилиндрического включения состава Si-Al в направлении $\langle 111\rangle$ под действием градиента температуры $\mathbf{G}(a)$ и модельное представление [11] формы сечения цилиндрического включения толщиной $l$, мигрирующего в направлении оси $0 x$, с двумя сингулярными (плоскими) и двумя несингулярными (криволинейными) участками межфазной границы $(b)$.

фазной энергии $\gamma_{\min } ; \gamma_{\max }-$ максимальное значение удельной межфазной энергии. В этом случае жидкое включение будет ограничено двумя сингулярными (атомно-гладкими, плоскими) и двумя несингулярными (атомно-шероховатыми, криволинейными) участками (рис. $1, b)$. Предполагается, что на атомно-шероховатых участках межфазные процессы протекают по нормальному механизму с пренебрежимо малым пересыщением (недосыщением), а на атомно-гладких участках — по дислокационному или зародышевому механизмам, описываемым зависимостями скорости границы $V$ от пересыщения (недосыщения) на ней $\Delta C[3,6]$ соответственно в виде:

$$
\begin{gathered}
V=\mu_{2 c(d)} \Delta C^{2}, \\
V=\mu_{3 c(d)} \exp \left(-\mu_{4 c(d)}|\Delta C|^{-1}\right),
\end{gathered}
$$

где $\mu_{2 c(d)}, \mu_{3 c(d)}, \mu_{4 c(d)}-$ кинетические коэффициенты, характеризующие степень затрудненности процессов кристаллизации (растворения).

\section{3. Результаты и их обсуждение}

На рис. 2 и 3 представлены результаты расчета зависимости скорости цилиндрического включения $V_{c i}$ от площади его сечения $S$ при разных значениях удельной межфазной энергии $\gamma_{\max }$, степени ее анизотропии $\eta=\gamma_{\max } / \gamma_{\min }$ и степени затрудненности межфазных процессов. Видно, что с уменьшением $\eta$ (приближением к 1) зависимость $V_{c i}(S)$ становится немонотонной, причем степень этой немонотонности выражена сильнее при относительно малых значениях удельной межфазной энергии $\gamma_{\max }$ (рис. 2) и относительно большой затрудненности процесса растворения (рис. 3). Такое изменение вида $V_{c i}(S)$ обусловлено разной степенью влияния капиллярных эффектов и межфазной кинетики на массоперенос в жидкой фазе. Влияние капиллярных эффектов, стремящихся придать сечению включения равновесную (симметричную) форму согласно зависимости (1), ослабляется с увеличением площади сечения и уменьшением $\gamma_{\max }$. В свою очередь межфазные ограничения на сингулярных участках, как отмечалось в [9-11], являются причиной деформации сечения мигрирующего (неравновесного) включения по отношению к его равновесной форме. При этом деформация тем сильнее, чем выше затрудненность межфазных процессов, то есть выше пересыщения (недосыщения) на сингулярных участках межфазной границы, обеспечивающих их движение с заданной скоростью. Поэтому форма малых включений, когда капиллярные эффекты существенны, лишь слегка отличается от равновесной, а скорость миграции возрастает с увеличением площади их сечения, так как при этом увеличивается толщина сечения (размер в направлении миграции), и, следовательно, общий перепад температур между сингулярными гранями. С дальнейшим увеличением площади $S$ капиллярные эффекты ослабляются, и влияние межфазной кинетики становится более заметным: степень деформации сечения включения возрастает вплоть до его уплощения (рис. 2, см. формы сечений включений у линий 6 и 7), а толщина сечения и, соответственно, перепад температур между сингулярными гранями перестают увеличиваться.

Интересно, что с увеличением площади сечения скорость включения и его толщина асимптотически приближаются к некоторым предельным значениям $\left(V_{\infty}\right.$ и $l_{\infty}$, соответственно), причем $V_{\infty}$ оказывается (рис. 2,3 , штрихпунктирные линии) меньше диффузионной скорости $V_{D}$, соответствующей случаю отсутствия межфазных ограничений (линии 1 на рис. 2 и 3) $[3,6]$. Предельные значения $V_{\infty}$ и $l_{\infty}$ могут быть рассчитаны на основе модели [11] с учетом того упрощающего факта, что с увеличением площади сечения включения кривизна криволинейного фронта кристаллизации в точке его стыка с плоским фронтом кристаллизации (при $x=0$, рис. $1, b)$ и степень понижения ликвидуса (за счет расширения плоского фронта растворения при $x=l$ ) стремятся к нулю. В этом случае задача о скорости цилиндрического включения становится аналогичной задаче о скорости плоской прослойки без учета кинетики кристаллизации, 


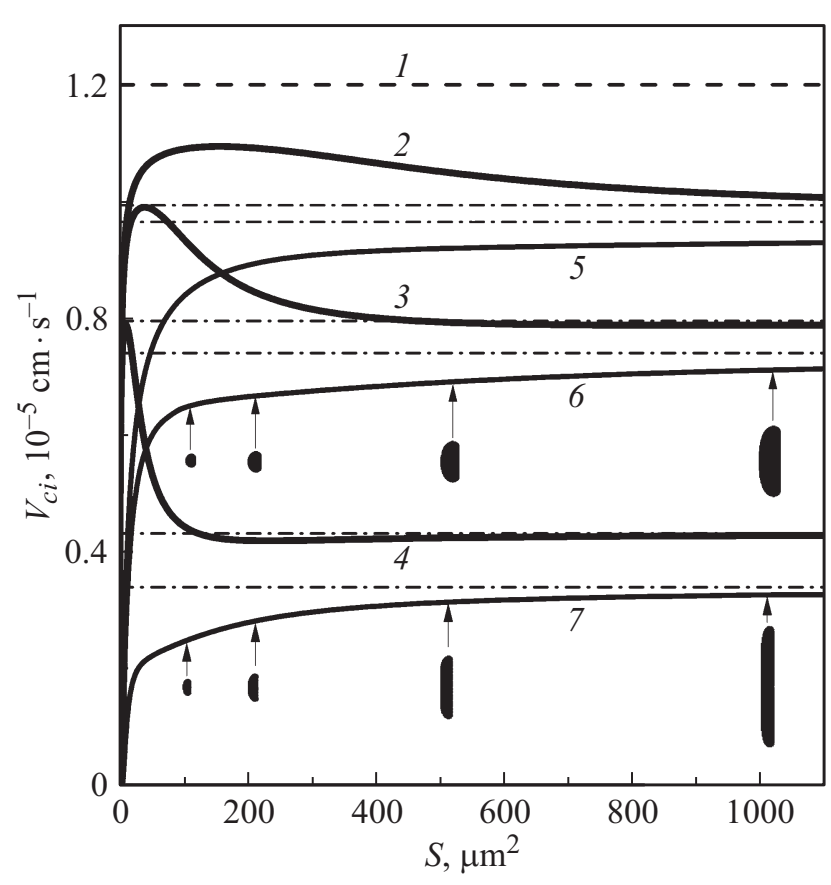

Рис. 2. Рассчитанные зависимости $V_{c i}(S)$ при разных значениях удельной межфазной энергии и степени ее анизотропии: $\gamma_{\max }=800$ (линии 2,5), 400 (линии 3,6), 200 (линии 4,7$) \mathrm{erg} / \mathrm{cm}^{2}$ и $\eta=1.05$ (линии $\left.2-4\right), 1.2$ (линии $5-7$ ) (зародышевый механизм: $\mu_{3 d(c)}=10^{5} \mathrm{~cm} / \mathrm{s} ; \mu_{4 d}=0.1$ at.\% и $\mu_{4 c}=0.05$ at.\%); линия 1 соответствует случаю отсутствия межфазных ограничений (диффузионная скорость $V_{D}$ ); предельные скорости $V_{\infty}$ отмечены штрихпунктирными линиями; формы сечений цилиндрических включений у линий 6 и 7 соответствуют скоростям и площадям сечений, указанным стрелками.

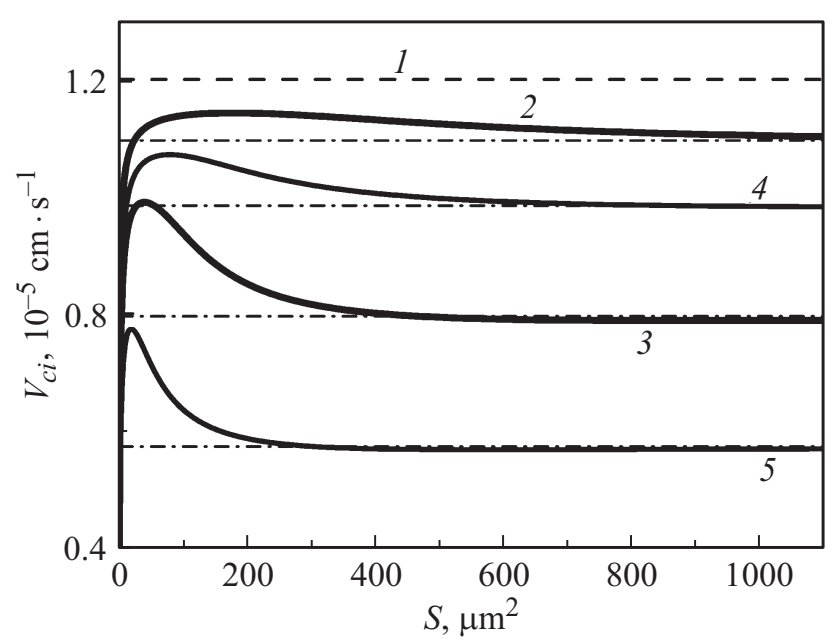

Рис. 3. Рассчитанные зависимости $V_{c i}(S)$ при разных механизмах межфазных процессов и степени их затрудненности: зародышевый механизм - $\mu_{3 d(c)}=10^{5} \mathrm{~cm} / \mathrm{s} ; \mu_{4 c}=0.05$ at.\%; $\mu_{4 d}=0.05$ (линия 2) и 0.1 at.\% (линия 3); дислокационный механизм - $\mu_{2 c}=1.0 \mathrm{~cm} \cdot \mathrm{s}^{-1} \cdot$ at. $\%^{-2} ; \mu_{2 d}=1.0$ (линия 4) и 0.2 (линия 5 ) $\mathrm{cm} \cdot \mathrm{s}^{-1} \cdot$ at. $\%^{-2}$; линия 1 соответствует случаю отсутствия межфазных ограничений (диффузионная скорость $\left.V_{D}\right)$; предельные скорости $V_{\infty}$ отмечены штрихпунктирными линиями; $\gamma_{\max }=400 \mathrm{erg} / \mathrm{cm}^{2}$ и $\eta=1.05$. решение которой, согласно модели [3], может быть записано в виде

$$
V_{\infty}=V_{D}\left[1-\Delta C_{\infty}\left(G l_{\infty} \frac{\partial C}{\partial T}\right)^{-1}\right],
$$

где $\Delta C_{\infty}=\frac{4 \gamma_{\min }}{l_{\infty}} \frac{\partial C}{\partial P}-$ предельное значение недосыщения на плоском фронте растворения [11]; $V_{D}=\frac{D G}{C^{S}-C^{L}} \frac{\partial C}{\partial T}-$ диффузионная скорость $[3,6] ; D-$ коэффициент взаимодиффузии компонентов жидкой фазы; $G$ - градиент температуры в жидкой фазе; $\frac{\partial C}{\partial T}-$ обратный наклон линии ликвидус; $C^{S}-C^{L}-$ разность концентраций вещества кристалла в твердой и жидкой фазах; $\frac{\partial C}{\partial P}-$ скорость изменения растворимости вещества кристалла при изменении давления.

С другой стороны, скорость $V_{\infty}$ должна также удовлетворять уравнениям $(2 a)$ или $(2 b)$ (в зависимости от выбранного механизма растворения) при недосыщении, равном $\Delta C_{\infty}$. Решая уравнение (3) совместно с $(2 a)$ или $(2 b)$, получим выражения для предельных скоростей в случае дислокационного и зародышевого механизмов соответственно

$$
\begin{gathered}
V_{\infty}=G \frac{\partial C}{\partial T}\left[\frac{C^{S}-C^{L}}{D}+\left(4 \mu_{2 d} \gamma_{\min } \frac{\partial C}{\partial P}\right)^{-1}\right]^{-1}, \quad(4 a) \\
V_{\infty}=\frac{D}{C^{S}-C^{L}}\left[G \frac{\partial C}{\partial T}-\frac{\mu_{4 d}^{2}}{4 \gamma_{\min } \ln ^{2}\left(\mu_{3 d} V_{\infty}^{-1}\right)}\left(\frac{\partial C}{\partial P}\right)^{-1}\right],
\end{gathered}
$$

(заметим, что выражение (4b) представляет собой трансцендентное уравнение, требующее численного решения).

Как следует из модели [3], в случае плоских прослоек жидкой фазы с увеличением ее объема (т.е. толщины прослоек $l$ ) влияние межфазной кинетики на массоперенос ослабляется, и скорость миграции приближается к диффузионной скорости $V_{D}$. В случае же цилиндрических включений при увеличении объема жидкой фазы (то есть площади поперечного сечения $S$ ), как видно из рис. 2,3 и выражений $(4 a),(4 b)$, предельные скорости включения меньше $V_{D}$, но тем ближе к ней, чем меньше затруднен процесс растворения $\left(\mu_{2 d} \rightarrow \infty\right.$, $\left.\mu_{3 d} \rightarrow \infty, \mu_{4 d} \rightarrow 0\right)$ и чем выше роль капиллярных эффектов $\left(\gamma_{\min } \rightarrow \infty\right)$.

Представляется интересным сравнить зависимости скорости цилиндрических включений $V_{c i}$ и плоских прослоек $V_{f l}$ от их толщины $l$ при прочих равных условиях. Из результатов расчетов, представленных на рис. 4, следует, что скорость цилиндрических включений (линии 2-7) выше скорости плоских прослоек той же толщины и при прочих равных условиях (линия 8) (заметим, что аналогичная закономерность отмечалась в [9], но для несколько иного вида анизотропии межфазной энергии чем (1)). При этом зависимость $V_{c i}(l)$ 


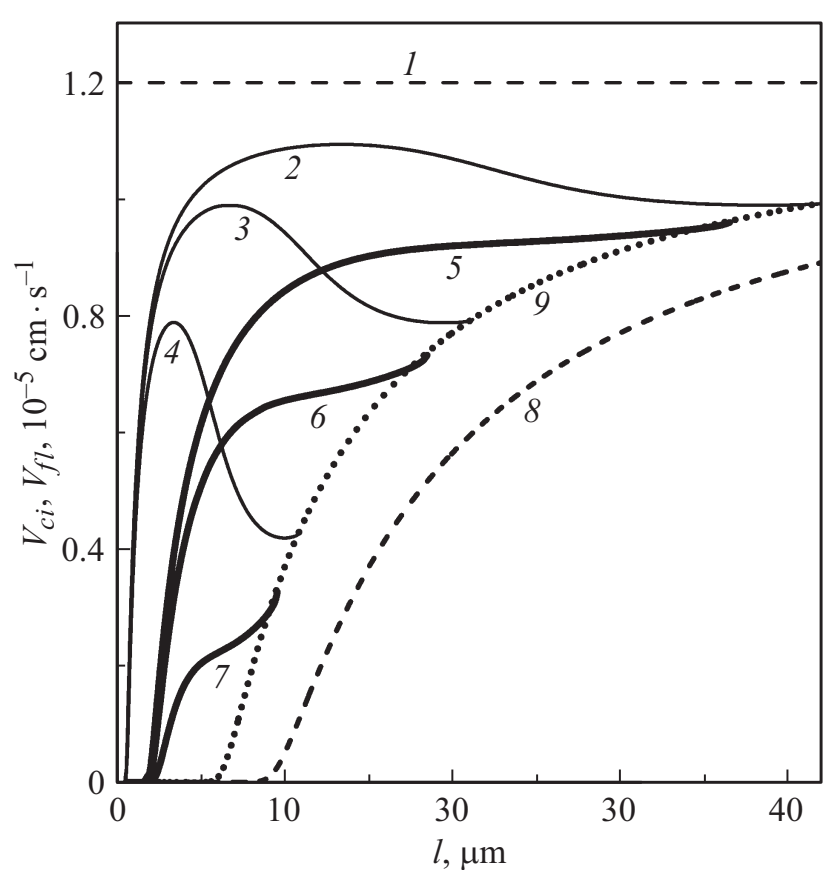

Рис. 4. Рассчитанные зависимости $V_{c i}(l)$ при $\gamma_{\max }=800$ (линии 2,5), 400 (линии 3,6), 200 (линии 4,7) $\mathrm{erg} / \mathrm{cm}^{2}$ и $\eta=1.05$ (линии 2-4), 1.2 (линии 5-7) (зародышевый механизм: $\mu_{3 d(c)}=10^{5} \mathrm{~cm} / \mathrm{s} ; \mu_{4 c}=0.05$ at. $\% ; \mu_{4 d}=0.1$ at.\%); линии 8 и 9 соответствуют зависимостям скорости плоских прослоек от их толщины $V_{f l}(l)$ в случае зародышевого механизма при $\mu_{3 d(c)}=10^{5} \mathrm{~cm} / \mathrm{s}, \mu_{4 d}=0.1$ at.\% и $\mu_{4 c}=0.05$ at.\% (линия 8 ), $\mu_{4 c} \rightarrow 0$ (линия 9); линия 1 соответствует случаю отсутствия межфазных ограничений (диффузионная скорость $V_{D}$ ).

(как и зависимость $V_{c i}(S)$ ) может иметь вид кривой с максимумом в отличие от случая плоских прослоек, для которых $V_{f l}(l)$ (рис. 4, линия 8) монотонно возрастающая функция, асимптотически приближающаяся при $l \rightarrow \infty$ к $V_{D}$ (рис. 4 , линия 1$)$.

Поскольку с ростом площади сечения цилиндрического включения его форма уплощается (рис. 2, см. формы сечений включений у линий 6 и 7), а скорость включения и его толщина стремятся к постоянным значениям $V_{\infty}$ и $l_{\infty}$, зависимость $V_{c i}(l)$ заканчивается в точке $\left(l_{\infty}, V_{\infty}\right)$. Как и следовало ожидать из предыдущего изложения, эта точка располагается на зависимости $V_{f l}(l)$ (рис. 4, линия 9) для плоских прослоек, построенной для случая, когда затруднен только процесс растворения (в той же степени, как и для цилиндрических включений), а кинетика кристаллизации не ограничивает массоперенос.

\section{4. Заключение}

Из анализа результатов проведенных расчетов следует, что зависимость скорости миграции цилиндрических включений от площади их сечения может быть немонотонной, в частности, убывать с увеличением объема жидкой фазы (площади сечения жидкого включения). Этот эффект выражен тем сильнее, чем меньше удельная межфазная энергия и степень ее анизотропии, а также чем выше затрудненность процесса растворения. Его следует учитывать при проведении экспериментов по термомиграции, поскольку цилиндрические включения с относительно большой площадью поперечного сечения в определенных условиях могут двигаться медленнее (вплоть до полной остановки), приняв при этом уплощенную форму, чем включения меныших размеров в тех же условиях. Скорость миграции цилиндрических включений с ростом площади их сечения не достигает значения диффузионной скорости, а асимптотически приближается к предельному значению, зависящему от удельной межфазной энергии плоского фронта растворения и затрудненности процесса растворения на нем.

\section{Благодарности}

Автор выражает благодарность Н.В. Лянгузову (ЮНЦ РАН) за электронную микроскопию образцов.

\section{Конфликт интересов}

Авторы заявляют, что у них нет конфликта интересов.

\section{Список литературы}

[1] Г.Г. Леммлейн. ДАН СССР 85, 325 (1952).

[2] W.G. Pfann. J. Met. 7, 961 (1955).

[3] W.A. Tiller. J. Appl. Phys. 34, 2757 (1963).

[4] T.R. Anthony, H.E. Cline. J. Appl. Phys. 42, 3380 (1971).

[5] Я.Е. Гегузин, М.А. Кривоглаз. Движение макроскопических включений в твердых телах. Металлургия, М. (1971). $344 \mathrm{c}$.

[6] В.Н. Лозовский, Л.С. Лунин, В.П. Попов. Зонная перекристаллизация градиентом температуры полупроводниковых материалов. Металлургия, М. (1987). 232 с.

[7] В.Ю. Гершанов, С.И. Гармашов. Нелинейные нестационарные эффекты в процессах массопереноса. Изд.-во Южного федерального ун-та, Ростов н/Д. (2014). 114 с.

[8] H.E. Cline, T.R. Anthony. J. Appl. Phys. 48, 5096 (1977).

[9] S.I. Garmashov, V.Yu. Gershanov. J. Cryst. Growth. 311, 413 (2009).

[10] В.Ю. Гершанов, С.И. Гармашов. ЖТФ 85, 61 (2015).

[11] С.И. Гармашов. Кристаллография 63, 827 (2018).

\section{Редактор т.Н. Василевская}

